\title{
Application of Flower-Like ZnO Nanorods Gas Sensor Detecting $\mathrm{SF}_{6}$ Decomposition Products
}

\author{
Shudi Peng, Gaolin Wu, Wei Song, and Qian Wang \\ Chongqing Electric Power Research Institute, Chongqing 401123, China \\ Correspondence should be addressed to Shudi Peng; psdzq@yahoo.cn
}

Received 21 November 2012; Accepted 3 January 2013

Academic Editor: Wen Zeng

Copyright (C) 2013 Shudi Peng et al. This is an open access article distributed under the Creative Commons Attribution License, which permits unrestricted use, distribution, and reproduction in any medium, provided the original work is properly cited.

Gas insulated switchgear (GIS) is an important electric power equipment in a substation, and its running state has a significant relationship with stability, security, and reliability of the whole electric power system. Detecting and analyzing the decomposition byproducts of sulfur hexafluoride gas $\left(\mathrm{SF}_{6}\right)$ is an effective method for GIS state assessment and fault diagnosis. This paper proposes a novel gas sensor based on flower-like $\mathrm{ZnO}$ nanorods to detect typical $\mathrm{SF}_{6}$ decompositions. Flower-like $\mathrm{ZnO}$ nanoparticles were synthesized via a simple hydrothermal method and characterized by X-ray powder diffraction and field-emission scanning electron microscopy, respectively. The gas sensor was fabricated with a planar-type structure and applied to detect $\mathrm{SF}_{6}$ decomposition products. It shows excellent sensing properties to $\mathrm{SO}_{2}, \mathrm{SOF}_{2}$, and $\mathrm{SO}_{2} \mathrm{~F}_{2}$ with rapid response and recovery time and long-term stability and repeatability. Moreover, the sensor shows a remarkable discrimination among $\mathrm{SO}_{2}, \mathrm{SOF}_{2}$, and $\mathrm{SO}_{2} \mathrm{~F}_{2}$ with high linearity, which makes the prepared sensor a good candidate and a wide application prospect detecting $\mathrm{SF}_{6}$ decomposition products in the future.

\section{Introduction}

Gas insulated switchgear (GIS) filled with pressurized sulfur hexafluoride gas $\left(\mathrm{SF}_{6}\right)$ is widely used in electric power system in recent decades with the advantages of small floor space, high stability and reliability, high-strength insulation, none smeary oil, lower maintenance cost, and so on [1-6]. Sulfur hexafluoride gas has excellent insulating performance and arc extinction function, and it can dramatically improve the insulation intensity when used as an insulating medium. So it is widely applied to GIS and other gas insulation equipments $[1,3]$. However, there exist some unavoidable insulating defects in the process of GIS design, manufacture, installation, and operation [4].

As an inert gas, pure $\mathrm{SF}_{6}$ is colorless, tasteless, nontoxic, and noninflammable, and its decomposition temperature is as high as $500^{\circ} \mathrm{C}$ [7]. Although $\mathrm{SF}_{6}$ is of great chemical inertness and the reliability of GIS is very high, inevitable insulating faults based on arc discharge, spark discharge, or partial discharge may occur due to the internal insulating defects. Researches both at home and aboard demonstrate that such internal insulation faults would cause $\mathrm{SF}_{6}$ gas to decompose, and generate several kinds of low-fluorine sulfides, such as $\mathrm{SF}_{4}, \mathrm{SF}_{3}$, and $\mathrm{SF}_{2}[2,4,5,8,9]$. If the $\mathrm{SF}_{6}$ in GIS is pure, the decomposed low-fluorine sulfides will reduce to $\mathrm{SF}_{6}$ fast with the decrease of operating temperature. Actually, it always contains a certain amount of impurities, such as air and water. Some low-fluorine sulfides are very active to react with trace moisture and oxygen and generate the compounds of $\mathrm{SOF}_{4}, \mathrm{SOF}_{2}, \mathrm{SO}_{2} \mathrm{~F}_{2}, \mathrm{SO}_{2}, \mathrm{HF}$, and so on. As the GIS insulating defects vary, the decomposed gas mixtures will be different. And the composition contents and decomposition rates are also various. Therefore, detecting and analyzing the decomposed chemical byproducts accurately can efficiently identity and diagnose fault type occurred in GIS.

At present, many methods [10-13] are used to detect the $\mathrm{SF}_{6}$ decomposition components in GIS, for instance, gas chromatography, gas detection tube, infrared absorption spectrometry, and semiconductor gas sensor. Gas chromatography [10] is mainly used for offline testing and it takes a quite long time. Gas detection tube [11] has no response to some decomposition components and its stability depends on environment condition. Infrared absorption spectrometry $[12,13]$ has cross-response on $\mathrm{SF}_{6}$ and cannot quantitatively 


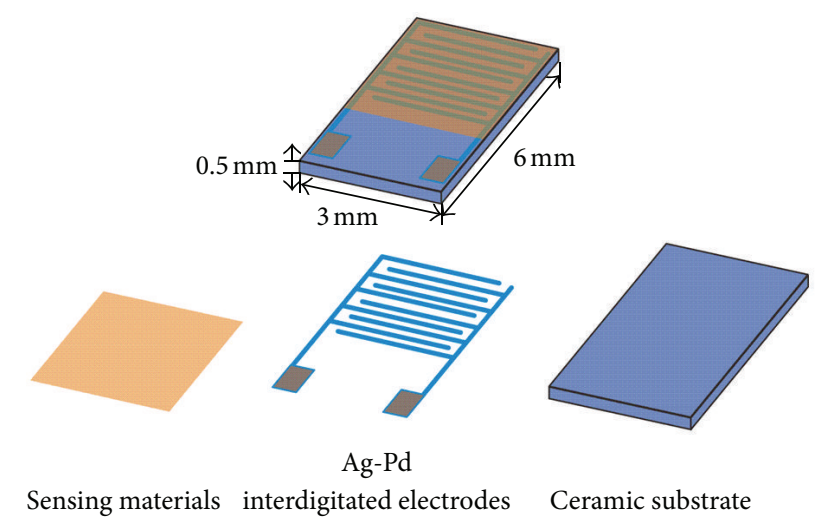

FIGURE 1: Schematic representation of planar $\mathrm{ZnO}$ gas sensor structure.

detect the decomposition components. In recent years, metal oxide semiconductor gas sensor based on $\mathrm{ZnO}$ [14], $\mathrm{SnO}_{2}$ [15], $\mathrm{TiO}_{2}$ [16], $\mathrm{Fe}_{2} \mathrm{O}_{3}$ [17], $\mathrm{WO}_{3}$ [18], or $\mathrm{In}_{2} \mathrm{O}_{3}$ [19] has been widely used for detecting and online monitoring target gas, owing to advantages of simple fabrication process, rapid response and recovery time, low maintenance cost, long service life, long-term stability and repeatability, and so on. With the development of nanotechnology, various gas sensors have been fabricated with small particle size and high surfaceto-volume ratio [20]. However, most of these gas sensors mainly focus on toxic gas [21,22], organic gas [23,24], carbon dioxide [25], hydrogen [26], and rare studies concerning the $\mathrm{SF}_{6}$ decompositions. Meanwhile, the cross-sensitivity among the decomposition components is tough, so investigating sensing properties especially selectivity is the most crucial issue for online monitoring $\mathrm{SF}_{6}$ decompositions.

In this work, we proposed a simple and effective hydrothermal synthesis route to prepare flower-like $\mathrm{ZnO}$ nanorods. X-ray powder diffraction (XRD) and fieldemission scanning electron microscopy (FESEM) were used to characterize the microstructures and morphologies of the prepared samples. Then a gas sensor based on the flower-like $\mathrm{ZnO}$ nanorods was fabricated, and its gas sensing properties against $\mathrm{SF}_{6}$ decompositions were investigated. Particularly, the study mainly focused on the sensing behaviors of the prepared sensor against $\mathrm{SOF}_{2}, \mathrm{SO}_{2} \mathrm{~F}_{2}$, and $\mathrm{SO}_{2}$, and its crosssensitivity was also demonstrated. The prepared sensor exhibited excellent gas response to different $\mathrm{SF}_{6}$ decompositions at different working temperature with high linearity, rapid response-recovery, and long-time stability and repeatability.

\section{Experimental}

2.1. Preparation and Characterization of $\mathrm{ZnO}$ Nanorods. Flower-like zinc oxide nanorods samples were successfully synthesized through a hydrothermal method using ammonium hydroxide $\left(\mathrm{NH}_{4} \mathrm{OH}, 28 \mathrm{wt} \% \mathrm{NH}_{3}\right.$ in $\left.\mathrm{H}_{2} \mathrm{O}\right)$ as the base source and zinc nitrate hexahydrate $\left(\mathrm{Zn}\left(\mathrm{NO}_{3}\right)_{2} \cdot 6 \mathrm{H}_{2} \mathrm{O}\right)$ as the source of $\mathrm{Zn}^{2+}$ ions. All chemicals were of analytical reagent grade and purchased from Beijing Chemicals Co., Ltd. In a typical synthesis process, an adequate amount of

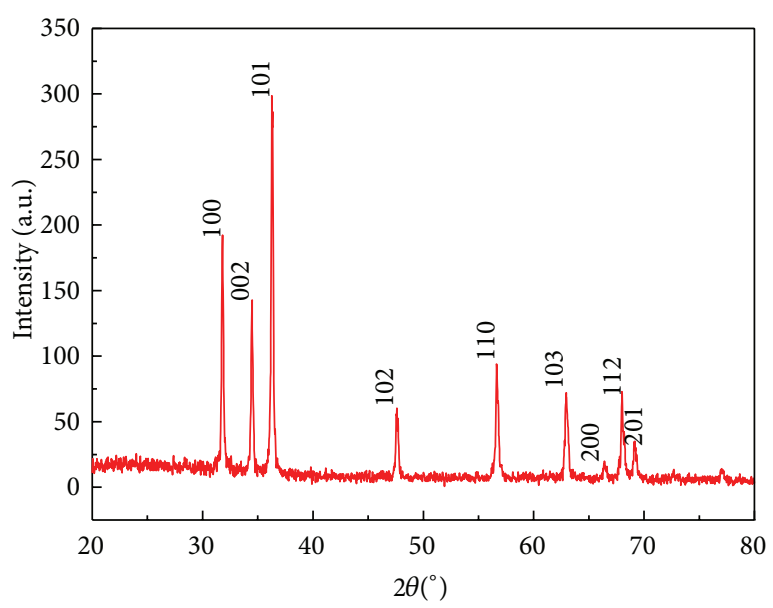

FIGURE 2: XRD patterns of the $\mathrm{ZnO}$ nanorods.

$\mathrm{Zn}\left(\mathrm{NO}_{3}\right)_{2} \cdot 6 \mathrm{H}_{2} \mathrm{O}$ was dissolved in deionized water (DI water) with a large beaker, and $\mathrm{NH}_{4} \mathrm{OH}$ was added slowly to the solution under intense magnetic stirring. The mixed solution was stirred for $30 \mathrm{~min}$ and then transferred into a sealed Teflon autoclave with $100 \mathrm{~mL}$ of inner volume and $80 \%$ of fill ratio. After $24 \mathrm{~h}$ reaction at $180^{\circ} \mathrm{C}$, the reactor was cooled to room temperature naturally. Subsequently, the prepared white products were centrifuged, washed two or three times with DI water and ethanol alternately, and dried at $80^{\circ} \mathrm{C}$ in air for further use.

XRD analysis was conducted on a Rigaku D/max-2500 $\mathrm{X}$-ray diffractometer with the $2 \theta$ range of $20-80^{\circ} \mathrm{C}$ at room temperature, and $\mathrm{Cu} K_{\alpha 1}$ as the source of X-ray at $40 \mathrm{kV}$, $40 \mathrm{~mA}$, and $\lambda=1.5418 \AA$. FESEM images were performed on a JEOL JEM-6700F microscope operating at 3 and $5 \mathrm{kV}$, respectively.

\subsection{Fabrication and Measurement of $\mathrm{ZnO}$ Sensor. $\mathrm{ZnO}$} nanorods gas sensor was fabricated based on a planar construction with a simple and convenient fabrication procedure. The scheme of the planar $\mathrm{ZnO}$ gas sensor structure was shown in Figure 1, where prepared planar $\mathrm{ZnO}$ nanorods gas sensor is constituted of planar ceramic substrate, Ag-Pd interdigitated electrodes, and sensing material. The length, width, and height of the planar ceramic substrate are suggested to be about 6,3 , and $0.5 \mathrm{~mm}$, respectively. There are five pairs of Ag-Pd interdigitated electrodes on planar ceramic substrate with both width and distance about $0.15 \mathrm{~mm}$. As-prepared samples were further ground into fine powder and mixed with diethanolamine and ethanol to form a paste with a weight ratio of $100: 10: 10$. It was subsequently screen printed onto the planar ceramic substrate to form a sensing film and the thickness was about $10 \mathrm{um}$ and then dried in air at $60^{\circ} \mathrm{C}$ for $5 \mathrm{~h}$. Finally, the sensor was further aged at an aging test chamber for $240 \mathrm{~h}$.

Gas sensing properties of the prepared planar $\mathrm{ZnO}$ gas sensor to $\mathrm{SF}_{6}$ decomposition byproducts were investigated using an intelligent gas detecting system. Targeted gases were 


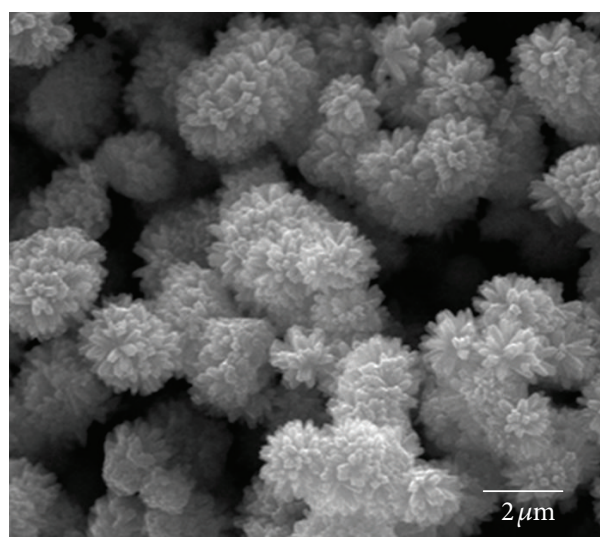

(a)

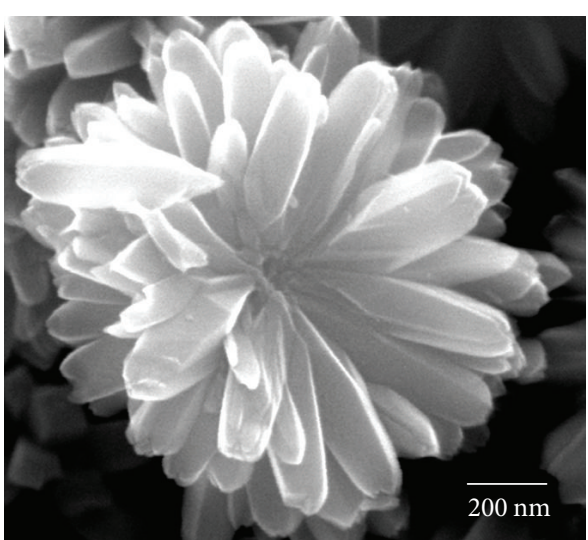

(b)

FIGURE 3: (a) Low-resolution FESEM image and (b) high-resolution FESEM image of the ZnO nanorods.

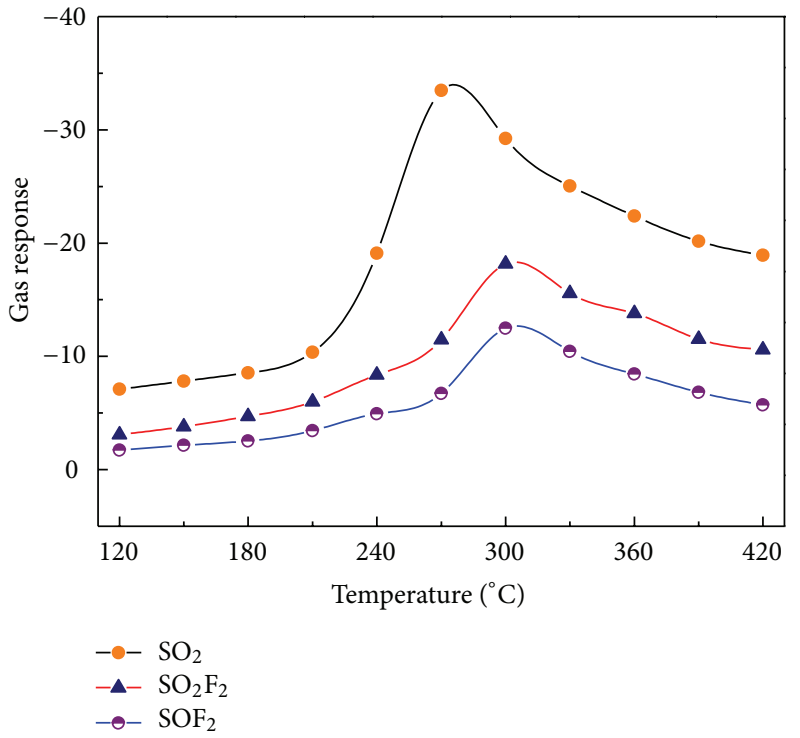

FIGURE 4: Gas response versus temperature curves to $50 \mu \mathrm{L} / \mathrm{L}$ of $\mathrm{SO}_{2}$, $\mathrm{SOF}_{2}$, and $\mathrm{SO}_{2} \mathrm{~F}_{2}$.

mixed with $\mathrm{N}_{2}$ by a dynamic gas distributing system which worked with high accuracy mass flow controllers and then injected into the gas sensing chamber. The concentration of detecting gas was controlled and detected by gas mass flow meter. The operating temperature of the gas sensor was controlled by varying current flow of the heater. And the surface temperature of the planar sensor was measured by a thermocouple in real time. When the testing sensor was preheated at $300^{\circ} \mathrm{C}$ for some time in air and the baseline of resistance was smooth and stable, we could start our gas sensing properties test.

Gas response was defined as the relative variation of the electrical resistance of the gas sensor: $S \%=\left(R-R_{0}\right) / R_{0} \times$ $100 \% . R$ is the resistance of flower-like $\mathrm{ZnO}$ nanorods gas sensor in target gas environment and $R_{0}$ being in pure air. The

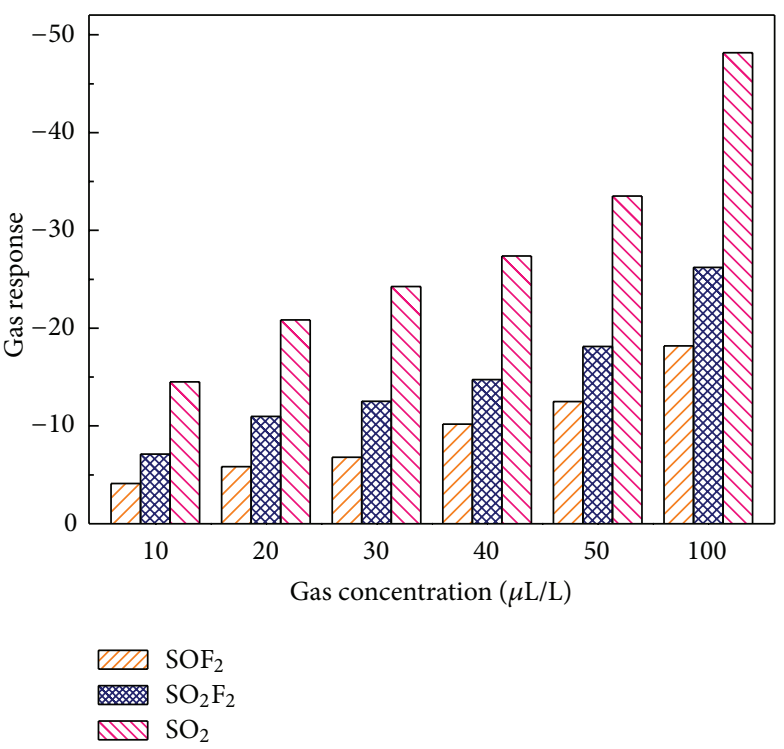

FIGURE 5: Gas response versus concentrations curves to $\mathrm{SO}_{2}, \mathrm{SOF}_{2}$, and $\mathrm{SO}_{2} \mathrm{~F}_{2}$.

response time was defined as the time taken by the sensor to achieve $90 \%$ of the total resistance change in the case of gas in or the recovery time in the case of gas out. All experiments were repeated several times to ensure the reproducibility and stability of the sensor.

\section{Results and Discussion}

3.1. Structure and Morphology. Figure 2 shows the XRD patterns of the as-prepared $\mathrm{ZnO}$ nanorods. All the diffraction peaks are consistent with the values in the standard card (JCPDS 36-1451) and can be indexed as typical wurtzite hexagonal $\mathrm{ZnO}$ crystal structure with lattice constants $a=$ $3.249 \AA$ and $c=5.206 \AA$. No other diffraction peaks from any impurities are detected. 


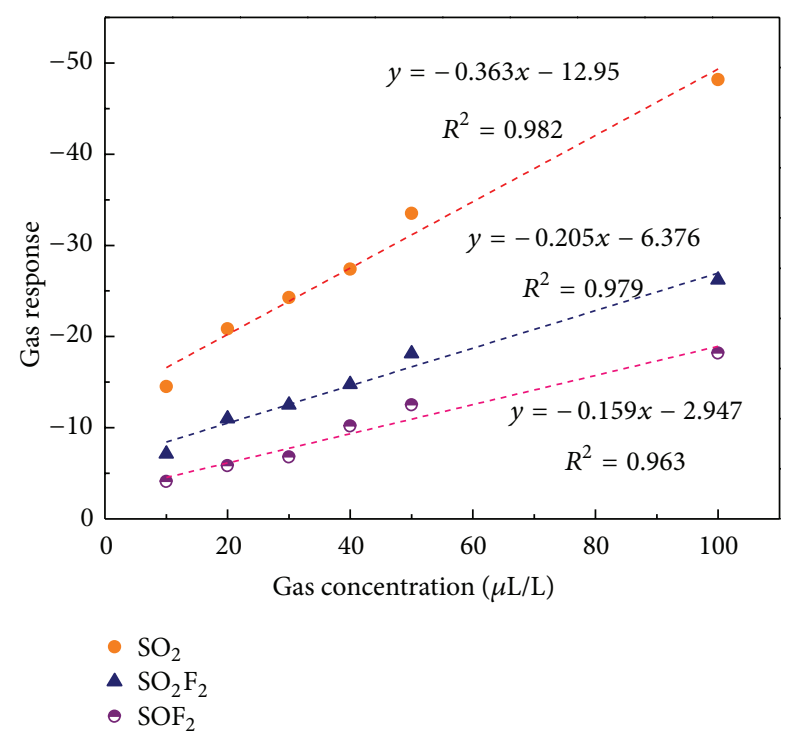

FIgURE 6: The linear calibration curves of $\mathrm{SO}_{2}, \mathrm{SOF}_{2}$, and $\mathrm{SO}_{2} \mathrm{~F}_{2}$.

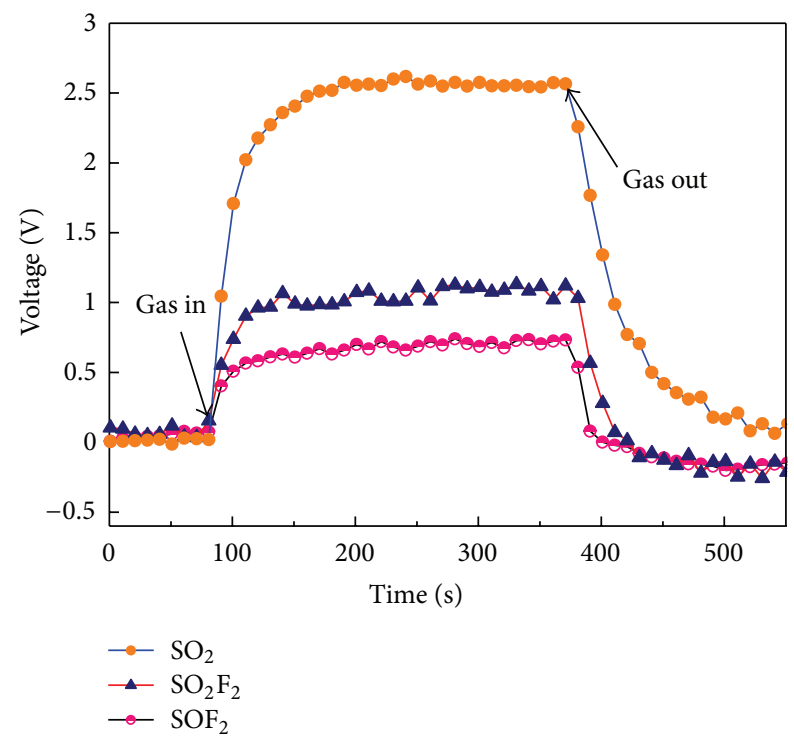

FIGURE 7: The response and recovery behaviors of the sensor to $10 \mu \mathrm{L} / \mathrm{L}$ of $\mathrm{SO}_{2}, \mathrm{SOF}_{2}$, and $\mathrm{SO}_{2} \mathrm{~F}_{2}$.

Figures 3(a) and 3(b) are typical low-resolution and high-resolution FESEM images of the prepared flower-like $\mathrm{ZnO}$ nanorods samples synthesized with the hydrothermal method. The nanoparticles have a high uniform flower-like bundle structure and self-assemble into flowers. The average length of $\mathrm{ZnO}$ nanorods is about $400 \mathrm{~nm}$ with an aspect ratio of $4: 1$.

3.2. Gas Sensing Properties and Sensing Mechanism. The gas sensing performances of metal oxide semiconductor gas sensor are dominantly influenced by working condition. Gas sensing experiments are performed with an intelligent gas detecting system at different operating temperatures to find out the optimum working temperature. Figure 4 shows the gas responses of the prepared flower-like $\mathrm{ZnO}$ nanorods gas sensor against $50 \mu \mathrm{L} / \mathrm{L}$ of $\mathrm{SF}_{6}$ compositions as a function of operating temperature, which ranges from $120^{\circ} \mathrm{C}$ to $420^{\circ} \mathrm{C}$. As seen in Figure 4, the measured gas response curves have a common change trend, in which gas response increases firstly with rising operating temperature and reaches the maximum, and then decreases with an continuous increase of the operating temperature.

This behavior can be understood by a dynamic equilibrium mechanism between gas adsorption and desorption process of gas molecule on the surface of $\mathrm{ZnO}$ or other similar semiconducting metal oxides. In the beginning, the rate of gas adsorption is much higher than that of desorption, and the amount of net adsorbed gas increases as the operating temperature rises. It would reach a saturated adsorption state and maintain a dynamic balance at the constant operating temperature. With a sequential increase of the operating temperature, the balance will be broken and it changes to a net desorption process, which ultimately results in a decreasing gas response. As shown in Figure 4, the optimal operating temperatures of the sensor to $50 \mu \mathrm{L} / \mathrm{L}$ of $\mathrm{SO}_{2}, \mathrm{SOF}_{2}$, and $\mathrm{SO}_{2} \mathrm{~F}_{2}$ are 250,300 , and $300^{\circ} \mathrm{C}$ with gas response of -33.44 , -12.47 , and -18.06 , respectively, which are applied in all the following investigations in this paper.

At their optimal operating temperatures, we performed the gas responses of the prepared plane flower-like $\mathrm{ZnO}$ gas sensor against different concentrations of $\mathrm{SO}_{2}, \mathrm{SOF}_{2}$, and $\mathrm{SO}_{2} \mathrm{~F}_{2}$. Figure 5 shows the relationship between gas responses and $10,20,30,40,50$, and $100 \mu \mathrm{L} / \mathrm{L}$ of $\mathrm{SO}_{2}, \mathrm{SOF}_{2}$, and $\mathrm{SO}_{2} \mathrm{~F}_{2}$, respectively. The gas response measured is manifested to persistently increase with a rising gas concentration. At the same level of gas concentration, the gas response values of the sensor to the three targeted gases decrease in the order of $\mathrm{SO}_{2}, \mathrm{SO}_{2} \mathrm{~F}_{2}$, and $\mathrm{SOF}_{2}$.

If the gas response curve is linear or quasilinear, the sensor can be applied to engineering application in practice. Therefore, based on the linear fitting tool in Origin software, linear characteristics of the prepared sensor to $\mathrm{SO}_{2}, \mathrm{SO}_{2} \mathrm{~F}_{2}$, and $\mathrm{SOF}_{2}$ were discussed. Figure 6 shows the linear calibration curves of the sensor to $\mathrm{SO}_{2}, \mathrm{SO}_{2} \mathrm{~F}_{2}$, and $\mathrm{SOF}_{2}$ with gas concentrations in the range of $10-100 \mu \mathrm{L} / \mathrm{L}$. As seen in Figure 6, all the three gas response curves meet highly linear with gas concentration, and the linear correlation coefficient $R^{2}$ for $\mathrm{SO}_{2}, \mathrm{SO}_{2} \mathrm{~F}_{2}$, and $\mathrm{SOF}_{2}$ is suggested to be about $0.982,0.979$, and 0.963 , respectively. Such a higher linear dependence indicates that our prepared flower-like $\mathrm{ZnO}$ gas sensor can be used as promising materials for detecting $\mathrm{SF}_{6}$ decompositions such as $\mathrm{SO}_{2}, \mathrm{SO}_{2} \mathrm{~F}_{2}$, and $\mathrm{SOF}_{2}$.

Response time and recovery time are other two key indicators to evaluate gas sensor performances. Figure 7 shows the response and recovery characteristic of the prepared sensor to $10 \mu \mathrm{L} / \mathrm{L}$ of $\mathrm{SO}_{2}, \mathrm{SO}_{2} \mathrm{~F}_{2}$, and $\mathrm{SOF}_{2}$ with the sensor working at its optimum operating temperature. As shown in Figure 7, the response times for $10 \mu \mathrm{L} / \mathrm{L}$ of $\mathrm{SO}_{2}, \mathrm{SO}_{2} \mathrm{~F}_{2}$, and $\mathrm{SOF}_{2}$ are about 21,13 , and $10 \mathrm{~s}$, and correspondingly the recovery times are about 45,32 , and $17 \mathrm{~s}$, respectively. 


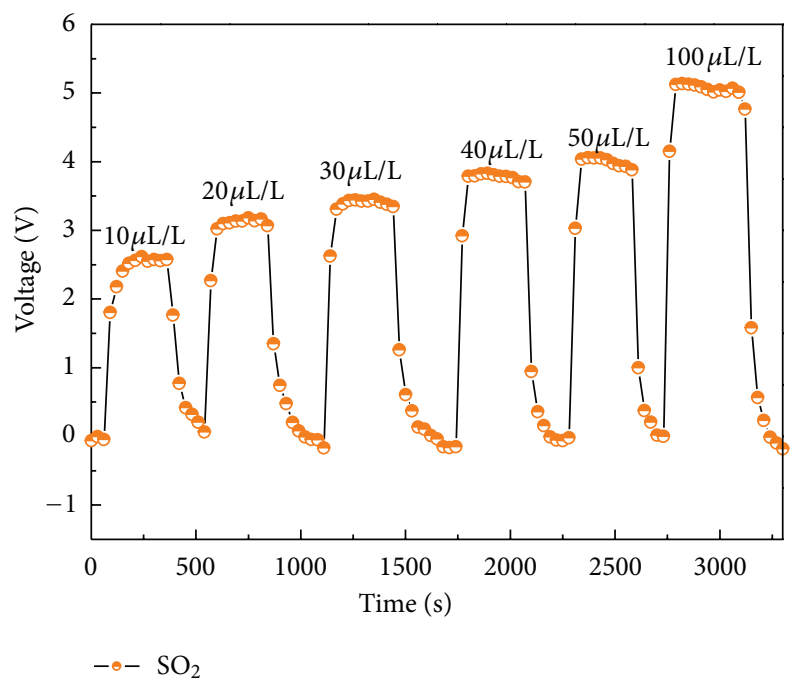

FIGURE 8: The response and recovery behaviors of the sensor to $\mathrm{SO}_{2}$.

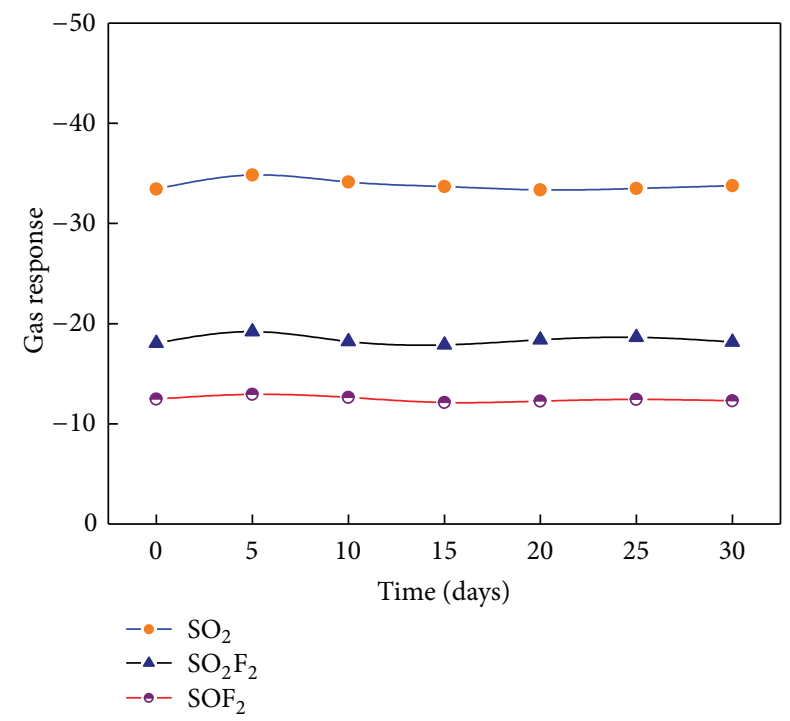

Figure 9: The stability and repeatability of the sensor against $50 \mu \mathrm{L} / \mathrm{L}$ of $\mathrm{SO}_{2}, \mathrm{SO}_{2} \mathrm{~F}_{2}$, and $\mathrm{SOF}_{2}$.

Such rapid response and recovery characteristic could be ascribed to the structure of the prepared flower-like sensor, which has a much bigger specific surface area than other conventional sensing structures, provides a larger adsorption area, and increases the amount of gas molecules adsorbed on the surface. Those advantages increase the rate of charge carriers and facilitate the movement of carriers through the barriers, consequently fast response and response property are observed.

The response and recovery behaviors versus $\mathrm{SO}_{2}$ with concentration at $10,20,30,40,50$, and $100 \mu \mathrm{L} / \mathrm{L}$ are shown in Figure 8 . With the concentration of detected gas increasing, the gas response amplitude increases apparently, nevertheless the response and recovery property changes slightly which indicates a very good and satisfying reproducibility of prepared sensor against the decompositions. Figure 9 shows the long-term stability and repeatability of the sensor against $50 \mu \mathrm{L} / \mathrm{L}$ of $\mathrm{SO}_{2}, \mathrm{SO}_{2} \mathrm{~F}_{2}$, and $\mathrm{SOF}_{2}$. One can clearly see in Figure 9 that the gas response changes slightly and keeps at a nearly constant value during the long experimental cycles, which confirms the excellent longtime stability and repeatability of the prepared flower-like $\mathrm{ZnO}$ nanorods gas sensor for detecting $\mathrm{SO}_{2}, \mathrm{SO}_{2} \mathrm{~F}_{2}$, and $\mathrm{SOF}_{2}$.

For most metal oxide semiconductor gas sensors such as zinc oxide, tin oxide, titanium oxide, ferric oxide, and indium oxide, the sensing properties are dominantly controlled by the change of electrical resistance [27], which is fundamentally attributed to the chemical adsorption and desorption process of gas molecules on sensing surface of the sensor.

It is well known to all that zinc oxide is a typical ntype semiconducting material and there exist many oxygen vacancies in the crystal lattices [28-30], where various kinds of oxygen could be adsorbed. The species of adsorbed oxygen are closely related to the ambient temperature [31]. At room temperature, oxygen is likely to be adsorbed on $\mathrm{ZnO}$ surface or grain boundaries with a typical physical adsorption mode. And it would turn into chemical adsorption by thermal excitation or electric excitation with certain energy.

As shown in Figure 10(a), oxygen would capture electrons and form a depletion region on the surface area, which results in a decrease in the concentration of charge carrier and electron mobility, thus gas sensor shows a higher electrical resistance. Figure 10(b) illustrates the gas sensing process of $\mathrm{SO}_{2}$ as an example exploring the gas sensing mechanism of the prepared sensor detecting $\mathrm{SF}_{6}$ decompositions. When flowerlike $\mathrm{ZnO}$ nanorods are reducing gas ambient at moderate temperature (such as in certain concentration of $\mathrm{SO}_{2}, \mathrm{SO}_{2} \mathrm{~F}_{2}$, and $\mathrm{SOF}_{2}$ ), the reducing gas reacts with chemical adsorbed oxygen, and then trapped electrons would be released back into $\mathrm{ZnO}$ surface. Electrons released from chemical adsorbed oxygen would reduce the height of barriers in the depletion region and increase the number of charge carriers [32, 33], which promotes the movements of charge carriers between conduction band and valence band and eventually increases the electrical conductivity of the sensor $[34,35]$.

With temperature rising, chemical adsorbed oxygen exists in various forms, namely, $\mathrm{O}_{2 \mathrm{ads}}{ }^{-}, \mathrm{O}_{\mathrm{ads}}{ }^{-}$, and $\mathrm{O}_{\mathrm{ads}}{ }^{2-}$, as shown in the following reaction equations:

$$
\begin{aligned}
& \mathrm{O}_{2 \mathrm{gas}} \longrightarrow \mathrm{O}_{2 \mathrm{ads}} \quad \mathrm{O}_{2 \mathrm{ads}}+\mathrm{e}^{-} \longrightarrow \mathrm{O}_{2 \mathrm{ads}}{ }^{-} \\
& \mathrm{O}_{2 \mathrm{ads}^{-}}{ }^{-}+\mathrm{e}^{-} \longrightarrow 2 \mathrm{O}_{\mathrm{ads}}{ }^{-} \quad \mathrm{O}_{\mathrm{ads}}{ }^{-}+\mathrm{e}^{-} \longrightarrow \mathrm{O}_{\mathrm{ads}}{ }^{2-}
\end{aligned}
$$

As mentioned above the state of adsorbed oxygen is mainly determined by the ambient temperature. At lower experimental temperatures, oxygen dominantly exists in the form of a "molecular ion" $\mathrm{O}_{2 \text { ads }}{ }^{-}$and transfers into "atomic ion" $\mathrm{O}_{\text {ads }}{ }^{-}$and $\mathrm{O}_{\text {ads }}{ }^{2-}$ with a further rising operating temperature. Experimental results indicate that the transition temperature for oxygen from "molecular ion" to "atomic ion" is about $450 \sim 500 \mathrm{~K}$. As performed in Figure 4, the optimum 


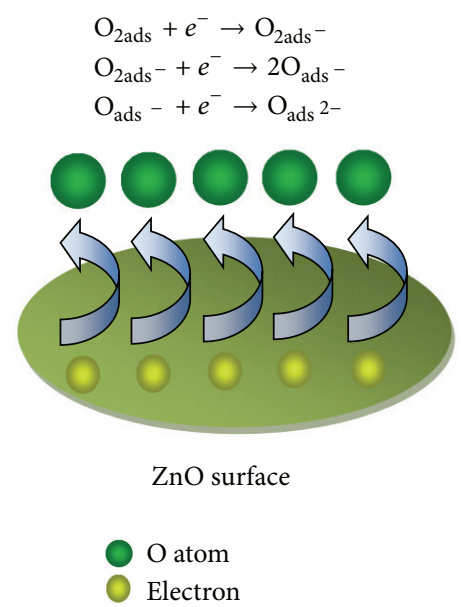

(a) Oxygen adsorbed on $\mathrm{ZnO}$ surface.

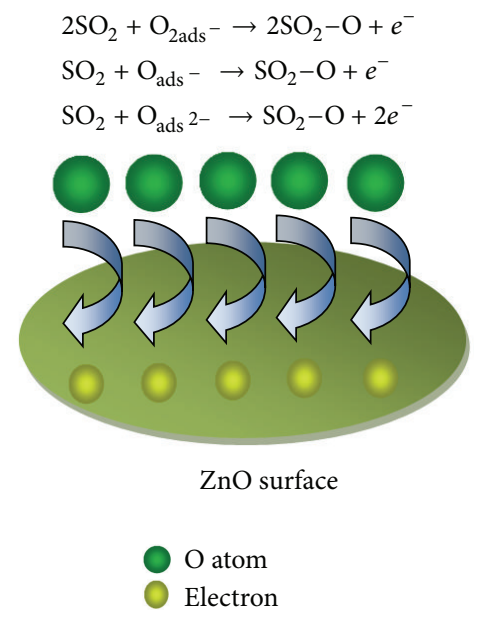

(b) $\mathrm{SO}_{2}$ gas sensing on $\mathrm{ZnO}$ surface $\mathrm{ZnO}$

FIGURE 10: Schematic plot illustrating the sensing mechanism of prepared sensor to $\mathrm{SO}_{2}$.

working temperatures for $\mathrm{SO}_{2}, \mathrm{SO}_{2} \mathrm{~F}_{2}$, and $\mathrm{SOF}_{2}$ are about 250,300 , and $300^{\circ} \mathrm{C}$, respectively. Thus, we draw a conclusion that the sensing behavior of the prepared sensor to $\mathrm{SO}_{2}$ gas may belong to the "molecular ion" reaction pattern, while it is an "atomic ion" gas response mode for $\mathrm{SO}_{2} \mathrm{~F}_{2}$ and $\mathrm{SOF}_{2}$.

\section{Conclusions}

In summary, Flower-like $\mathrm{ZnO}$ nanorods have been successfully synthesized and characterized by XRD and FESEM. The optimum operating temperatures of the prepared sensor to $\mathrm{SO}_{2}, \mathrm{SO}_{2} \mathrm{~F}_{2}$, and $\mathrm{SOF}_{2}$ are about 250,300 , and $300^{\circ} \mathrm{C}$. The response (recovery) time of the sensor to $10 \mu \mathrm{L} / \mathrm{L}$ of $\mathrm{SO}_{2}$, $\mathrm{SO}_{2} \mathrm{~F}_{2}$, and $\mathrm{SOF}_{2}$ is 21 (45), 13 (32), and 10 (17) s, respectively. Especially, the flower-like $\mathrm{ZnO}$ nanorods gas sensor shows high linearity to $\mathrm{SO}_{2}, \mathrm{SO}_{2} \mathrm{~F}_{2}$, and $\mathrm{SOF}_{2}$ at the range of $10-$ $100 \mu \mathrm{L} / \mathrm{L}$ with excellent linear correlation coefficient $R^{2}$ at $0.982,0.979$, and 0.963 , separately. These findings demonstrate that our prepared flower-like $\mathrm{ZnO}$ nanorods have some excellent potential advantages for using as gas sensors to detect and online monitor the $\mathrm{SF}_{6}$ decompositions such as $\mathrm{SO}_{2}, \mathrm{SOF}_{2}$, and $\mathrm{SO}_{2} \mathrm{~F}_{2}$ in practice, although further studies are still needed.

\section{References}

[1] J. Tang, F. Liu, X. X. Zhang, Q. H. Meng, and J. B. Zhou, "Partial discharge recognition through an analysis of $\mathrm{SF}_{6}$ decomposition products Part 1: decomposition characteristics of $\mathrm{SF}_{6}$ under four different partial discharges," IEEE Transactions on Dielectrics and Electrical Insulation, vol. 19, no. 1, pp. 29-36, 2012.

[2] M. Shih, W. J. Lee, and C. Y. Chen, "Decomposition of $\mathrm{SF}_{6}$ and $\mathrm{H}_{2} \mathrm{~S}$ mixture in radio frequency plasma environment," Industrial and Engineering Chemistry Research, vol. 42, no. 13, pp. 2906-2912, 2003.

[3] J. Tang, F. Liu, X. X. Zhang, Q. H. Meng, and J. G. Tao, "Partial discharge recognition through an analysis of $\mathrm{SF}_{6}$ decomposition products part 2: feature extraction and decision tree-based pattern recognition," IEEE Transactions on Dielectrics and Electrical Insulation, vol. 19, no. 1, pp. 37-44, 2012.

[4] R. J. Van Brunt and J. T. Herron, "Fundamental processes of $\mathrm{SF}_{6}$ decomposition and oxidation in glow and corona discharges," IEEE Transactions on Electrical Insulation, vol. 25, no. 1, pp. 7594, 1990.

[5] M. Shih, W. J. Lee, C. H. Tsai, P. J. Tsai, and C. Y. Chen, "Decomposition of $\mathrm{SF}_{6}$ in an RF plasma environment," Journal of the Air and Waste Management Association, vol. 52, no. 11, pp. 1274-1280, 2002.

[6] I. Sauers, H. W. Ellis, and L. G. Christophorou, "Neutral decomposition products in spark breakdown of $\mathrm{SF}_{6}$," IEEE Transactions on Electrical Insulation, vol. EI-21, no. 2, pp. 111120, 1986.

[7] W. T. Tsai, "The decomposition products of sulfur hexafluoride $\left(\mathrm{SF}_{6}\right)$ : reviews of environmental and health risk analysis," Journal of Fluorine Chemistry, vol. 128, no. 11, pp. 1345-1352, 2007.

[8] L. Vial, A. M. Casanovas, I. Coll, and J. Casanovas, "Decomposition products from negative and $50 \mathrm{~Hz}$ ac corona discharges in compressed $\mathrm{SF}_{6}$ and $\mathrm{SF}_{6} / \mathrm{N}_{2}(10: 90)$ mixtures. Effect of water vapour added to the gas," Journal of Physics D, vol. 32, no. 14, pp. 1681-1692, 1999.

[9] C. T. Dervos and P. Vassiliou, "Sulfur hexafluoride ( $\left.\mathrm{SF}_{6}\right)$ : Global environmental effects and toxic byproduct formation," Journal of the Air and Waste Management Association, vol. 50, no. 1, pp. 137-141, 2000.

[10] E. Duffour, "Molecular dynamic simulations of the collision between copper ions, $\mathrm{SF}_{6}$ molecules and a polyethylene surface: a study of decomposition products and an evaluation of the selfdiffusion coefficients," Macromolecular Theory and Simulations, vol. 19, no. 2-3, pp. 88-99, 2010.

[11] J. I. Baumbach, P. Pilzecker, and E. Trindade, "Monitoring of circuit breakers using ion mobility spectrometry to detect $\mathrm{SF}_{6}$ decomposition," International Journal for Ion Mobility Spectrometry, vol. 2, no. 1, pp. 35-39, 1999.

[12] R. Kurte, C. Beyer, H. M. Heise, and D. Klockow, "Application of infrared spectroscopy to monitoring gas insulated high-voltage equipment: electrode material-dependent $\mathrm{SF}_{6}$ decomposition," 
Analytical and Bioanalytical Chemistry, vol. 373, no. 7, pp. 639646, 2002.

[13] W. Ding, R. Hayashi, K. Ochi et al., "Analysis of PD-generated $\mathrm{SF}_{6}$ decomposition gases adsorbed on carbon nanotubes," IEEE Transactions on Dielectrics and Electrical Insulation, vol. 13, no. 6, pp. 1200-1207, 2006.

[14] J. Singh, A. Mukherjee, S. K. Sengupta, J. Im, G. W. Peterson, and J. E. Whitten, "Sulfur dioxide and nitrogen dioxide adsorption on zinc oxide and zirconium hydroxide nanoparticles and the effect on photoluminescence," Applied Surface Science, vol. 258, no. 15, pp. 5778-5785, 2012.

[15] B. Wang, L. F. Zhu, Y. H. Yang, N. S. Xu, and G. W. Yang, "Fabrication of a $\mathrm{SnO}_{2}$ nanowire gas sensor and sensor performance for hydrogen," Journal of Physical Chemistry C, vol. 112, no. 17, pp. 6643-6647, 2008.

[16] J. Gong, Y. Li, Z. Hu, Z. Zhou, and Y. Deng, "Ultrasensitive $\mathrm{NH}_{3}$ gas sensor from polyaniline nanograin enchased $\mathrm{TiO}_{2}$ fibers," Journal of Physical Chemistry C, vol. 114, no. 21, pp. 9970-9974, 2010.

[17] X. Liu, J. Zhang, X. Guo, S. Wu, and S. Wang, "Porous $\alpha$ $\mathrm{Fe}_{2} \mathrm{O}_{3}$ decorated by Au nanoparticles and their enhanced sensor performance," Nanotechnology, vol. 21, no. 9, Article ID 095501, 2010.

[18] B. Cao, J. Chen, X. Tang, and W. Zhou, "Growth of monoclinic $\mathrm{WO}_{3}$ nanowire array for highly sensitive $\mathrm{NO}_{2}$ detection," Journal of Materials Chemistry, vol. 19, no. 16, pp. 2323-2327, 2009.

[19] S. E. Moon, H. Y. Lee, J. Park et al., "Low power consumption and high sensitivity carbon monoxide gas sensor using indium oxide nanowire," Journal of Nanoscience and Nanotechnology, vol. 10, no. 5, pp. 3189-3192, 2010.

[20] W. Zeng, T. Liu, Z. Wang, S. Tsukimoto, M. Saito, and Y. Ikuhara, "Selective detection of formaldehyde gas using a Cd-Doped $\mathrm{TiO}_{2}-\mathrm{SnO}_{2}$ sensor," Sensors, vol. 9, no. 11, pp. 9029-9038, 2009.

[21] M. Chen, Z. Wang, D. Han, F. Gu, and G. Guo, "Porous ZnO polygonal nanoflakes: synthesis, use in high-sensitivity $\mathrm{NO}_{2}$ gas sensor, and proposed mechanism of gas sensing," Journal of Physical Chemistry C, vol. 115, no. 26, pp. 12763-12773, 2011.

[22] E. Oh, H. Y. Choi, S. H. Jung et al., "High-performance $\mathrm{NO}_{2}$ gas sensor based on $\mathrm{ZnO}$ nanorod grown by ultrasonic irradiation," Sensors and Actuators B, vol. 141, no. 1, pp. 239-243, 2009.

[23] K. Zheng, L. Gu, D. Sun, X. Mo, and G. Chen, “The properties of ethanol gas sensor based on Ti doped $\mathrm{ZnO}$ nanotetrapods," Materials Science and Engineering B, vol. 166, no. 1, pp. 104-107, 2010.

[24] A. Wei, L.-H. Pan, X.-C. Dong, and W. Huang, "Roomtemperature $\mathrm{NH}_{3}$ gas sensor based on hydrothermally grown ZnO nanorods," Chinese Physics Letters, vol. 28, no. 8, pp. 702706, 2011.

[25] C. Wen, Y. Ju, W. Li et al., "Carbon dioxide gas sensor using SAW device based on $\mathrm{ZnO}$ film," Applied Mechanics and Materials, vol. 135-136, pp. 347-352, 2012.

[26] O. Lupan, G. Chai, and L. Chow, "Novel hydrogen gas sensor based on single $\mathrm{ZnO}$ nanorod," Microelectronic Engineering, vol. 85, no. 11, pp. 2220-2225, 2008.

[27] W. Zeng, T. Liu, and Z. Wang, "Enhanced gas sensing properties by $\mathrm{SnO}_{2}$ nanosphere functionalized $\mathrm{TiO}_{2}$ nanobelts," Journal of Materials Chemistry, vol. 22, no. 8, pp. 3544-3548, 2012.

[28] J. Kim and K. Yong, "Mechanism study of $\mathrm{ZnO}$ nanorod-bundle sensors for $\mathrm{H}_{2} \mathrm{~S}$ gas sensing," Journal of Physical Chemistry $\mathrm{C}$, vol. 115, no. 15, pp. 7218-7224, 2011.
[29] D. Velasco-Arias, D. Díaz, P. Santiago-Jacinto, G. RodríguezGattorno, A. Vázquez-Olmos, and S. E. Castillo-Blum, "Direct interaction of colloidal nanostructured $\mathrm{ZnO}$ and $\mathrm{SnO}_{2}$ with $\mathrm{NO}$ and $\mathrm{SO}_{2}$," Journal of Nanoscience and Nanotechnology, vol. 8, no. 12, pp. 6389-6397, 2008.

[30] Q. Qi, T. Zhang, Q. Yu et al., "Properties of humidity sensing $\mathrm{ZnO}$ nanorods-base sensor fabricated by screen-printing," Sensors and Actuators B, vol. 133, no. 2, pp. 638-643, 2008.

[31] M.-W. Ahn, K.-S. Park, J.-H. Heo et al., "Gas sensing properties of defect-controlled ZnO-nanowire gas sensor," Applied Physics Letters, vol. 93, no. 26, Article ID 263103, 2008.

[32] M. W. Ahn, K. S. Park, J. H. Heo, D. W. Kim, K. J. Choi, and J. G. Park, "On-chip fabrication of $\mathrm{ZnO}$-nanowire gas sensor with high gas sensitivity," Sensors and Actuators B, vol. 138, no. 1, pp. 168-173, 2009.

[33] J. Zhang, S. Wang, M. Xu et al., "Hierarchically porous $\mathrm{ZnO}$ architectures for gas sensor application," Crystal Growth and Design, vol. 9, no. 8, pp. 3532-3537, 2009.

[34] Z. Yuan, X. Jiaqiang, X. Qun, L. Hui, P. Qingyi, and X. Pengcheng, "Brush-like hierarchical zno nanostructures: synthesis, photoluminescence and gas sensor properties," Journal of Physical Chemistry C, vol. 113, no. 9, pp. 3430-3435, 2009.

[35] J. Zhang, X. Liu, S. Wu, B. Cao, and S. Zheng, "One-pot synthesis of Au-supported $\mathrm{ZnO}$ nanoplates with enhanced gas sensor performance," Sensors and Actuators B, vol. 169, pp. 61-66, 2012. 

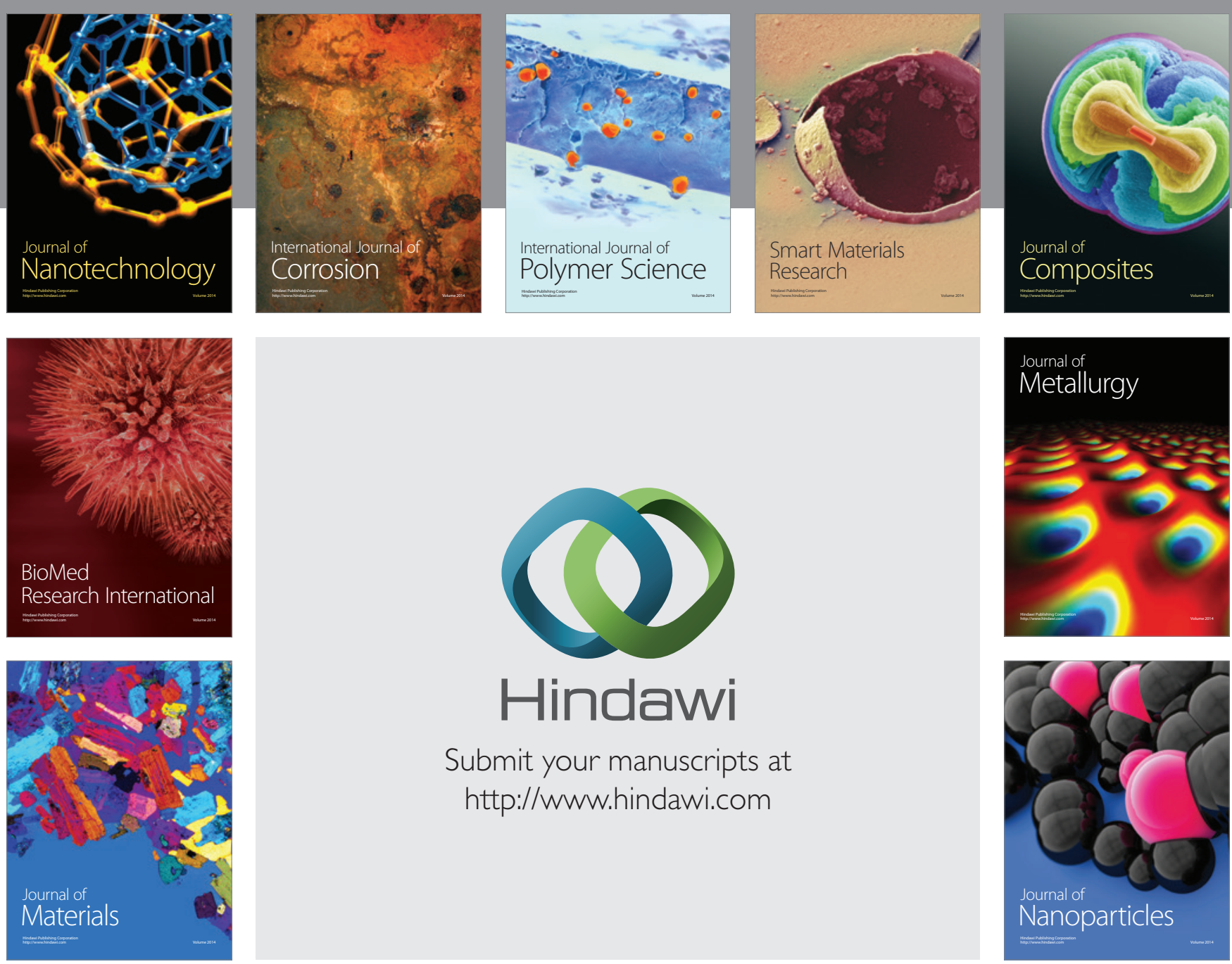

Submit your manuscripts at http://www.hindawi.com
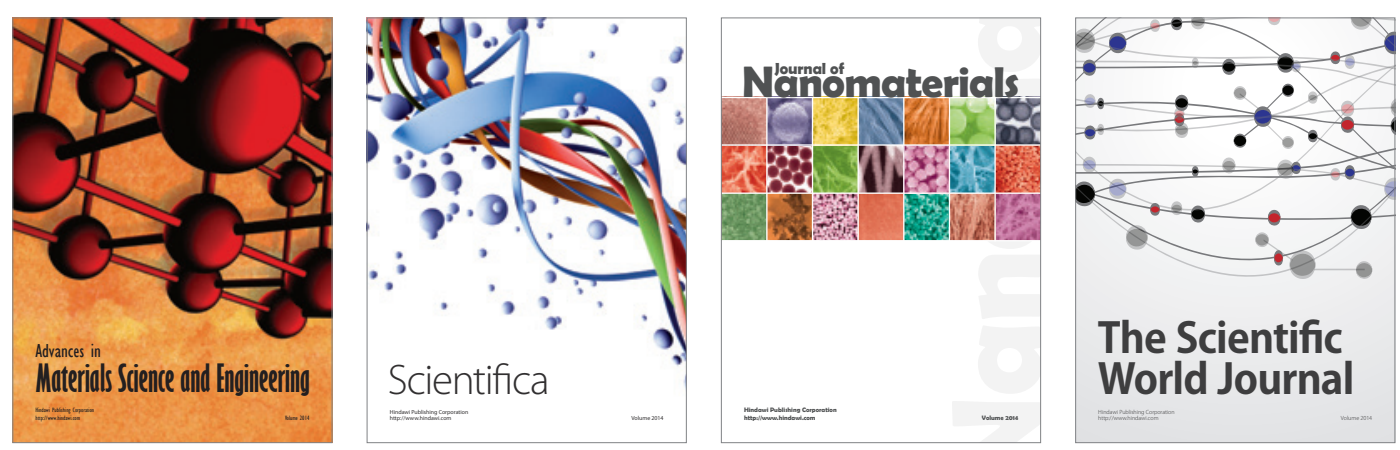

\section{The Scientific World Journal}
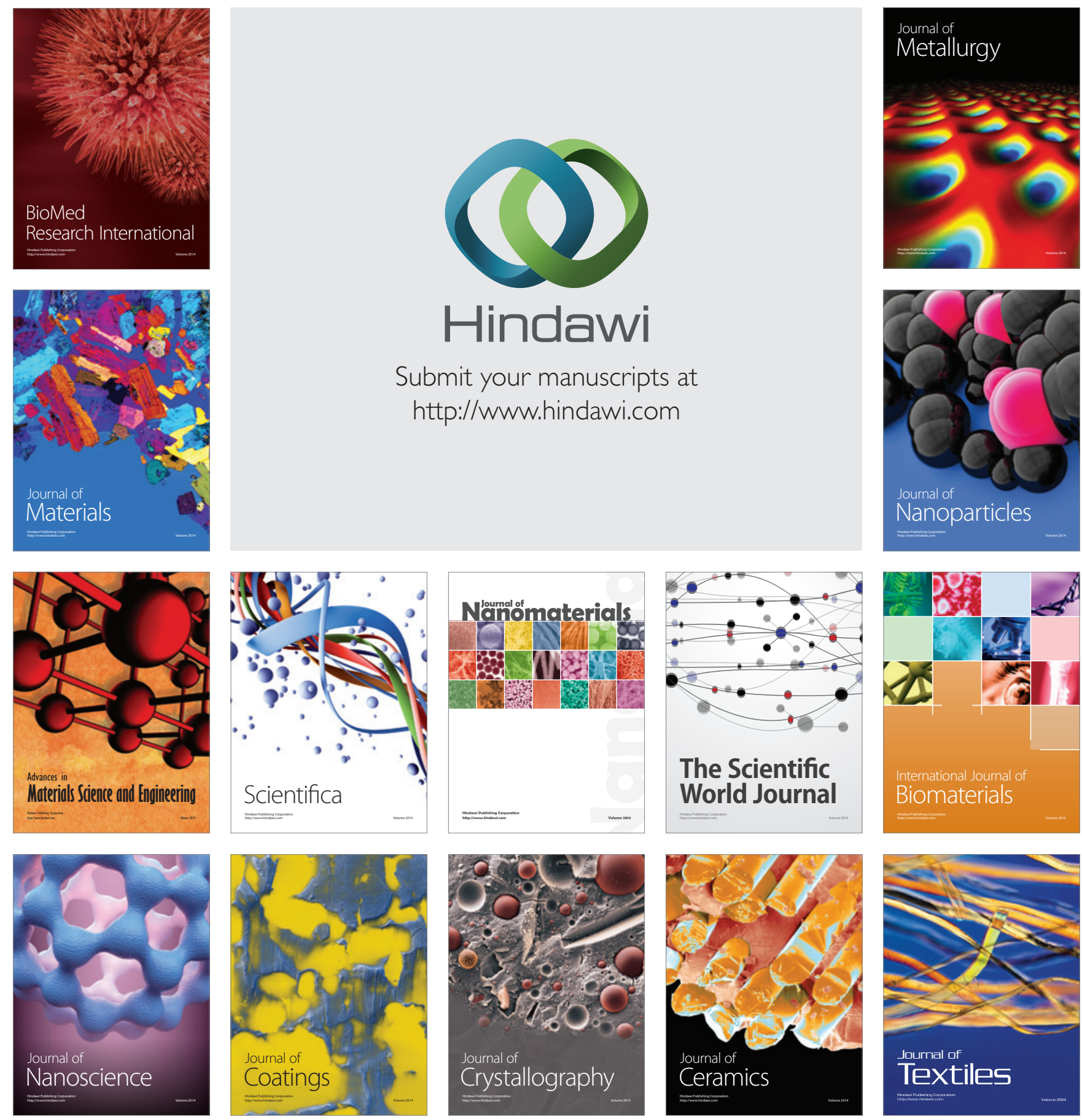